\title{
Atomic-Scale STEM-EELS Characterization of the Chemistry of Structural Defects and Interfaces in Energy-Related Materials
}

Q.M. Ramasse ${ }^{1}$, F. Azough ${ }^{2}$, R. Freer ${ }^{2}$, D.M. Kepaptsoglou ${ }^{1}$, R. Mainz ${ }^{3}$, A. Weber ${ }^{3}$, D. Abou-Ras ${ }^{3}$, E. Simsek $^{4}$ and P.A. van Aken ${ }^{4}$

${ }^{1}$ SuperSTEM Laboratory, STFC Daresbury Campus, Keckwick Lane, Daresbury WA4 4AD, UK

2 School of Materials, University of Manchester, Manchester M13 9PL, UK

${ }^{3}$ Helmholtz Zentrum Berlin, Hahn Meitner Platz 1, 14109 Berlin, Germany

${ }^{4}$ Max Planck Institute for Intelligent Systems, Heisenbergstr. 3, 70569 Stuttgart, Germany

The physical properties of functional energy materials often depend on their precise chemistry and atomic arrangements at the sub-angstrom scale, and minute changes in composition or structure can dramatically alter the materials' properties. These effects are often too local to be fully understood through bulk characterization methods and advances in analytical scanning transmission electron microscopy (STEM), with atomic-resolution Z-contrast imaging and true routine spectroscopic chemical mapping, have made STEM-EELS a near-ubiquitous tool when attempting to gain a better understanding of the defects' influence on materials performance.

For instance, $\mathrm{Cu}(\mathrm{In}, \mathrm{Ga}) \mathrm{Se}_{2}$ (CIGSe) based chalcopyrite-type solar cells fabricated with multi-stage co-evaporation show high power-conversion efficiencies of more than $20 \%$ [1]. However, in many cases, the efficiencies obtained with CIGSe-based devices fall well behind this value. This is in particular true in low-temperatures processes without a so-called $\mathrm{Cu}$-rich stage [2]. The fundamental reasons for this efficiency loss as well as the limitations for further efficiency increase are not fully understood, although chemical modulations at or in the vicinity of structural defects and grain boundaries are widely thought to play an important role. HR-STEM and EELS analyses show striking chemical characteristics for a number of observed defects in the Cu-poor CIGSe thin films. The chemistry of twin boundaries along the $\{112\}$ planes of the chalcopyrite structure, which are very frequent in these samples, is entirely identical to the rest of the grains, with a homogeneous distribution of all the elements. By contrast, $\mathrm{Cu}$ enrichment in combination with $\mathrm{In}$ and Se depletion were systematically observed at random grain boundaries and within complex defects closely related to stacking faults: figure $1 . \mathrm{Cu}_{\mathrm{x}} \mathrm{Se}$-rich channels seem to form immediately outside (but not within) dislocation cores. These results suggest that such defects must play a crucial role for the photovoltaic properties of the material as it is indeed known that $\mathrm{Cu}$ depletion causes a slight bang gap increase in this system [3].

Furthermore, when these imaging and chemical mapping techniques are combined with advanced statistical methods it is possible to correlate variations in chemical distribution with accurately measured small local atomic displacements. This approach has proved particularly successful when studying series of thermoelectric complex oxide ceramics such as the $(1-\mathrm{x}) \mathrm{SrTiO}_{3}-\mathrm{xLa}_{1 / 3} \mathrm{NbO}_{3}$ system in which the ionic conductivity and thermoelectric behaviour appears to be controlled at least in part by vacancy ordering depending on the La concentration [4]. Here a correlative analysis of HAADF and EELS map intensities over large datasets distinguishes between two categories of A cation sites in this perovskite structure. 


\section{References:}

[1] P. Jackson, D. Hariskos, E. Lotter et al., Prog. Photovoltaics 19 (2011), p. 894.

[2] R. Caballero, C.A. Kauffmann, V. Efimova et al., Prog. Photovolt.: Res. and Appl. 21 (2013), p. 30.

[3] S.-H. Han, F.S. Hasoon, H.A. Al-Thani et al., J. Phys. Chem. Sol. 66 (2005), p. 1895.

[4] A Kinaci, C Sevik and T Cagin, Phys.Rev. B 82 (2011), p. 155114-1.

[5] C.J. Howard, J. Sol. State Chem. 117 (2004), p. 155114-1.

[6] SuperSTEM is funded by the UK Engineering and Physical Sciences Research Council (EPSRC).
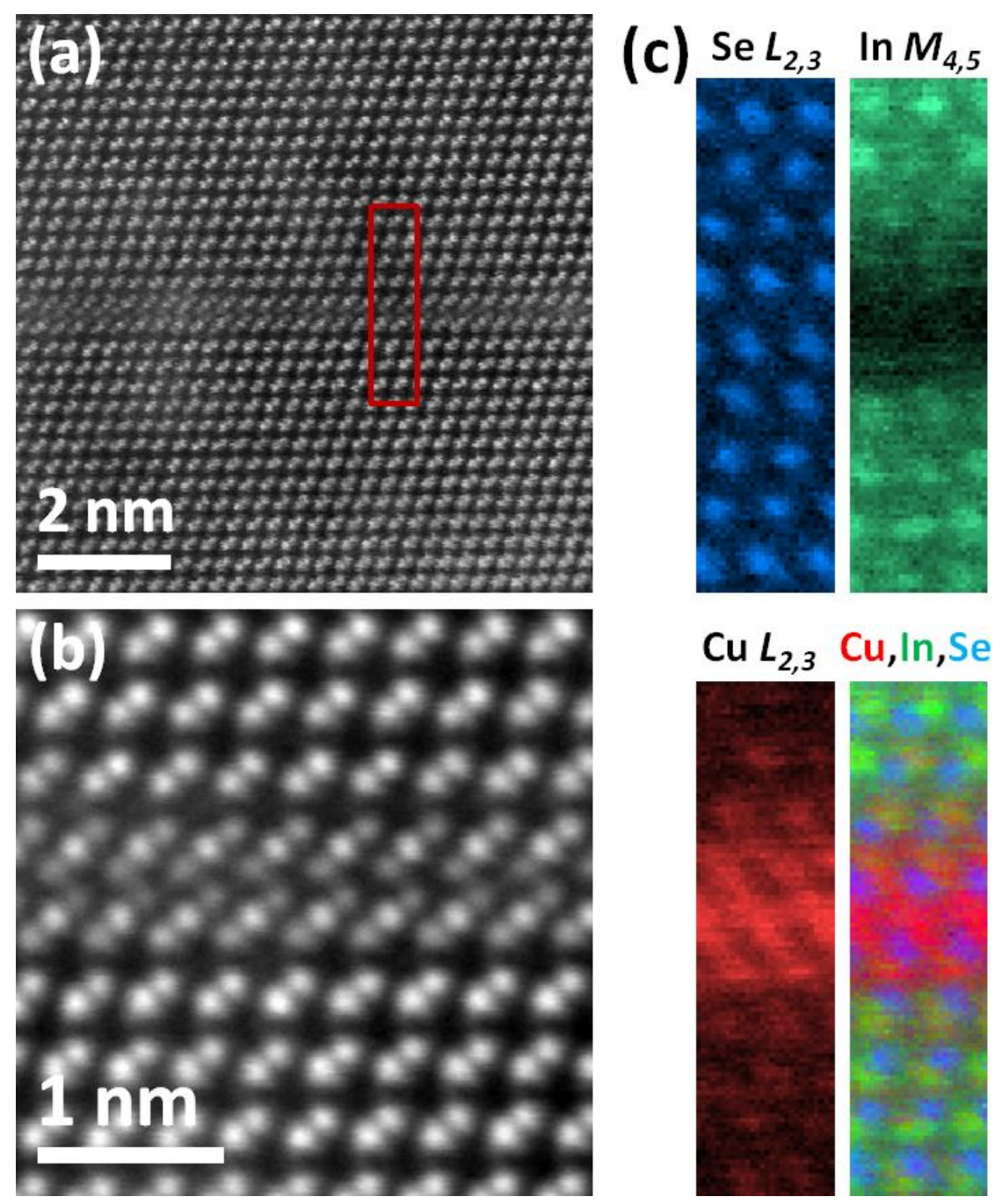

Figure 1. (a) HAADF image of a complex defect in $\mathrm{Cu}(\mathrm{In}, \mathrm{Ga}) \mathrm{Se}_{2}$. (b)Averaged sequential imaging can be used to obtain very high signal-to-noise of these defects: here 50 consecutive frames are aligned and averaged to reveal with high precision the atomic arrangement at this stracking fault-type defect. (c) EELS chemical mapping shows a very strong $\mathrm{Cu}$ enrichment and simultaneous In depletion at the defect. Note the continuity of the Se sub-lattice. 